\title{
DO WE USE METHYLATION OF NFATC1 AND FOS GENES AS A BIOMARKER FOR POSTMENOPAUSAL OSTEOPOROSIS?
}

\author{
Kalkan $\mathrm{R}^{1, *}$, Tosun $\mathrm{O}^{2}$
}

*Corresponding Author: Associate Professor Rasime Kalkan, Department of Medical Genetics, Faculty of Medicine, Near East University, Near East Boulevard, Nicosia, Cyprus, 99138. Tel: +903-92223-6464. Fax:+903-92-223-6461. E-mail: rasime.kalkan@neu.edu.tr, kalkanr@yahoo.com

\begin{abstract}
Genetic and epigenetic factors have an important role during the development of osteoporosis. Receptor activator of nuclear factor- $\kappa \mathrm{B}(\mathrm{NF}-\kappa \mathrm{B})(\mathrm{RANK}) /$ receptor activator of NF- $\kappa$ B ligand (RANKL) pathway is important for the bone remodeling, and NFATC1 and FOS are the downtargets of this pathway. Here, we report methylation status of NFATC1 and FOS genes in post- and premenopausal women. In this study, 30 premenopausal and 35 postmenopausal women were included. Methylation sensitive-high resolution melting (MS-HRM) analysis was used for identification of NFATC1 and FOS genes methylation. The NFATC1 gene was methylated in 11 of the 35 postmenopausal women, and the FOS gene was methylated in six of the postmenopausal women $(p>0.005)$. Here, we found statistically significant association between unmethylation of the NFATC1 gene and postmenopausal status. This result explains the epigenetic regulation of osteoclasts during the menopausal transition, and for the first time, our results can be used for epigenetic explanation of postmenopausal osteoporosis in the literature. However, the limited number of studies in this field makes our results crucial. Our results showed great value of epigenetic profiles of postmenopausal women.
\end{abstract}

Keywords: FOS; NFATC1; Methylation sensitivehigh resolution melting (MS-HRM); Methylation; Postmenopause; Premenopause

\footnotetext{
${ }^{1}$ Department of Medical Genetics, Faculty of Medicine, Near East University, Nicosia, Cyprus

${ }^{2}$ Department of Biostatistics, Faculty of Medicine, Near East University, Nicosia, Cyprus
}

\section{INTRODUCTION}

The nuclear factor of activated T-cell gene family contains five members; which are NFATC1, NFATC2, NFATC3, NFATC4 and NFATC5 [1]. This gene family is regulated by the calcium signaling pathway [1], which plays an important role during the regulation of the different systems; for instance, immune system [2], skeletal system and circulatory system [2-4]. NFATCl regulates osteoclast-specific genes for osteoclast differentiation, such as TRAP, cathepsin K, calcitonin receptor and FOS [5-8]. The osteoclast differentiation pathway was under control of RANKL [receptor activator of nuclear factor- $\mathrm{B}$ (NF$\kappa \mathrm{B})$ ligand], $N F-\kappa B$ and $F O S$ signaling [9]. The RANK/ RANKL is an important pathway for bone remodeling [10], which activates FOS and triggers NFATC1 expression [8]. The interaction between FOS and osteoclast differentiation was observed in mice, and researchers showed osteoporosis development in FOS knockout mice [5,11].

Due to the genetic and epigenetic factors, osteoporosis has been reported as a multifactorial disease [12]. Several association studies had been done but the etiology and molecular mechanism of the disease is not clearly explained. During the menopause, the deficiency of estrogen causes increased level of the follicle-stimulating hormone (FSH) which accelerates bone loss [13]. Differential gene expression of bone cells has been done to solve this complex interaction $[14,15]$. Liu et al. [16] used circulating monocytes for genome wide differential gene expression analysis in osteoporotic and non osteoporotic postmenopausal Caucasian females, and they identified differential gene expression in circulating monocytes. In general studies that analyzed the effect of estrogens, corticosteroids and oxidative stress on osteoblast cells $[15,17]$ showed the gene expression profile or polymorphisms of different genes in premenopausal and primary osteoporosis women $[15,18]$. 
To the best of our knowledge, there are no epigenetic studies of NFATC1 and FOS genes in postmenopausal women. In this study, we aim to analyze methylation status of NFATC1 and FOS genes in post- and premenopausal women and show their association with menopausal osteoporosis. Based on the current literature, this is the first epigenetic study on postmenopausal women that shows postmenopausal osteoporosis and NFATC1 gene interaction.

\section{MATERIALS AND METHODS}

The study protocol was approved by the Near East University Local Ethics Committee [YDU/2016/42-352], Nicosia, Cyprus. Written informed consent was obtained from all participants. Genomic DNA was extracted from blood samples according to the AllPrep DNA/RNA/Protein (Qiagen GmbH, Hilden, Germany) isolation kit, and its quantity was measured using a NanoDrop ND-1000 Spec-trophotometer (Thermo Fisher Scientific, Waltham, MA USA) [19].

Subjects. In this study, the subjects were divided into two groups. One group consisted of 35 postmenopausal women with a mean age of $56.7 \pm 4.9$, and the other group consisted of 30 premenopausal women with a mean age of $33.5 \pm 6.9$. None of the participants had hypertension, liver, kidney, diabetes, thyroid or cardiovascular disease. Medical therapy history was obtained for all subjects. They did not receive any medications or participate in any dietary or exercise program during the study. All participants were genetically unrelated postmenopausal females. Subjects with abnormal menopause, took medications such as anxiolytics, anti-depressants and/ or exogenous hormone. Women who had serious disease or mental retardation, smoking, alcohol usage and had weight loss therapy, food allergies, heart disease history, insulin-dependent diabetes mellitus type 2 (T2DM), kidney disease or liver disease, were excluded. Individuals who had been in postmenopause for a minimum of 1 year were recruited.

Determination of NFATC1 and FOS Methylation Status. Bisulfite modification was applied according to the manufacturer's protocol (EpiTect Bisulfite Kit; Qiagen
$\mathrm{GmbH})$ and $1.3 \mu \mathrm{g}$ DNA was used for bisulfite treatment reaction. The Rotor Gene Q (Qiagen $\mathrm{GmbH}$ ) was used for methylation sensitive-high resolution melting (MS-HRM) analysis to detect the methylation status of NFATC1 and FOS genes, and universal methylated and unmethylated DNA (EpiTect Control DNA Set, Cat No. 59568; Qiagen $\mathrm{GmbH}$ ) was also used as a control in each reaction. The primers for each were designed according to the EpiTect ${ }^{\circledR}$ HRM $^{\text {TM }}$ PCR Handbook (Qiagen GmbH) [19].

Statistical analysis. The statistical analyses and their associations with patient characteristics were performed by Pearson's $\chi^{2}$ test and two-tailed Fisher's exact test. Calculations were performed using the Statistical Package for Social Science (SPSS) version 16.0 software (SPSS Inc., Chicago, IL, USA), and a $p$ level of 0.05 was considered to be statistically significant.

\section{RESULTS}

The mean age of 30 premenopause patients was 33.5 \pm 6.9 years and the mean age of 35 postmenopause patients was $56.7 \pm 4.9$ years. There were no significant differences between the premenopausal and postmenopausal group in bone mineral density (BMD) $(p>0.005)$.

The NFATC1 promotor methylation was detected in 11 of the 35 postmenopausal women (31.4\%) (Figure 1) and unmethylated in 24 of the 35 postmenopausal women (68.8\%). The NFATC1 promotor methylation was detected in 19 of the $30(63.3 \%)$ control sample (premenopausal women) and unmethylated in 11 of the 30 control sample (36.7\%). Here, we found statistically significant correlation between postmenopause and unmethylation of NFATC1 promotor $(p=0.010)$.

The FOS promotor methylation was detected in six of the 35 (17.1\%) postmenopausal women (Figure 2) and unmethylated in 29 of the 35 postmenopausal women $(82.9 \%)(p>0.005)$. The FOS promotor methylation was detected in five of the $30(16.7 \%)$ control sample (premenopausal women) and unmethylated in 25 of the 30 control sample (83.3\%). Moreover, there was no statistically significant association identified between menopause and methylation status $(p>0.05)$ (Table 1$)$.

Table 1. Methylation status of NFATC1 and FOS in post- and premenopausal women.

\begin{tabular}{|l|c|c|c|}
\hline Parameters & $\begin{array}{c}\text { Postmenopausal } \\
\boldsymbol{n}(\mathbf{\%})\end{array}$ & $\begin{array}{c}\text { Premenopausal } \\
\boldsymbol{n}(\mathbf{\%})\end{array}$ & $\boldsymbol{p}$ Value \\
\hline NFATC1 methylated & $11(31.4)$ & $19(63.3)$ & 0.010 \\
\hline NFATC1 unmethylated & $24(68.8)$ & $11(36.7)$ & $>0.050$ \\
\hline FOS methylated & $6(17.1)$ & $5(16.7)$ & \\
\hline FOS unmethylated & $29(82.9)$ & $25(83.3)$ & \\
\hline
\end{tabular}




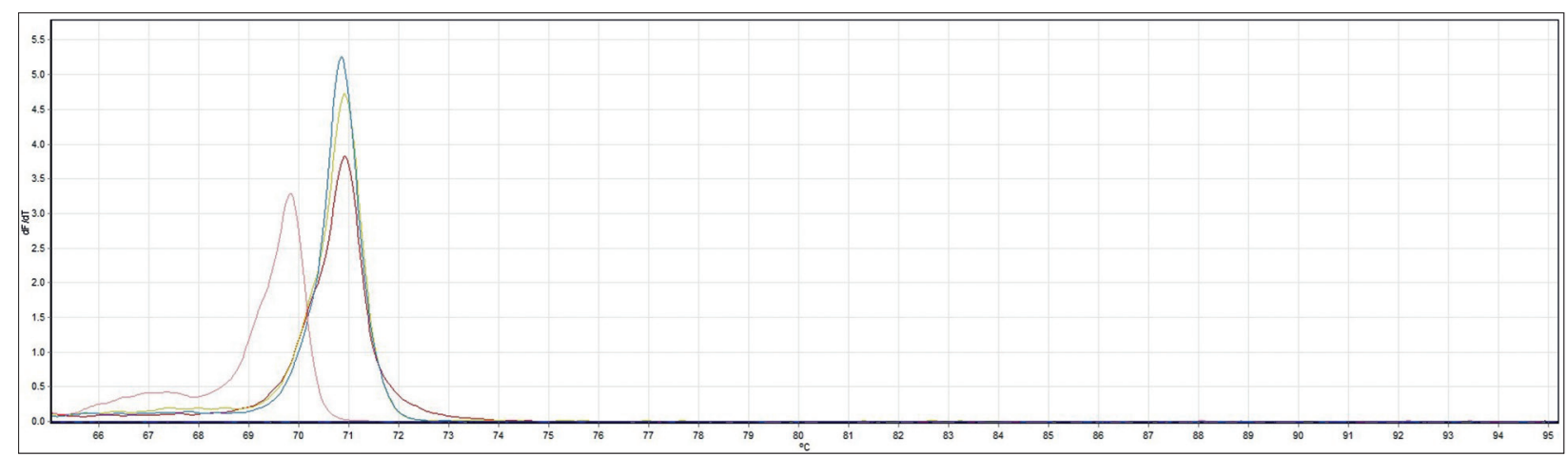

Figure 1. Methylated NFATC1 patients. The NFATC1 unmethylated control was orange, the methylated control was red. Patients 41 and 34 were methylated.

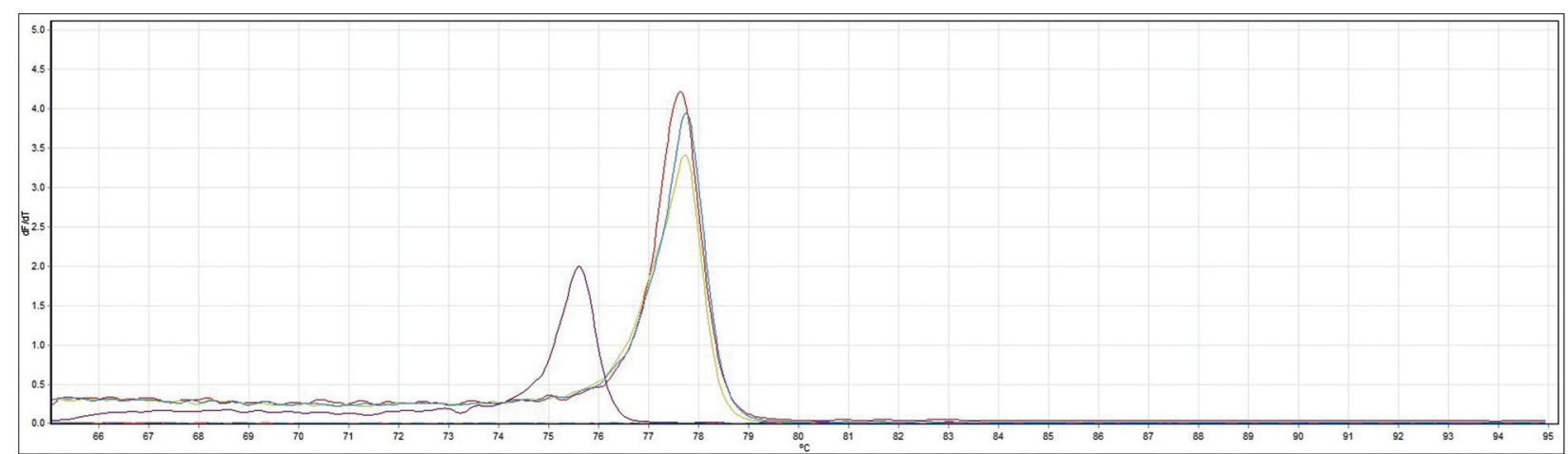

Figure 2. Methylated FOS patients. The FOS unmethylated control was purple, the methylated control was red. Patients 8 and 19 were methylated.

\section{DISCUSSION}

The RAS signaling pathway is important for the osteoclast survival and triggers activation of downstream genes [20]. NFATC1 and FOS are the downtargets of the RAS pathway, which have an important role during osteoclastogenesis [20]. NFATC1 regulates osteoclast-specific genes during the regulation of osteoclast differentiation and FOS is one of the genes which NFATC1 triggers their expression [5-8]. Raggatt and Partridge [21], demonstrated that NFATC1 is one of the important transcription factors during the differentiation of osteoclast precursors.

Decreased level of estrogen triggers several physiological and hormonal problems. Postmenopausal osteoporosis is one of the consequences of decreased level of estrogen [22,23]. Gavali et al. [24] demonstrated the effect of estrogen on osteoclast differentiation and they showed that lack of estrogen causes failure of apoptosis and inhibits activation of NFATC1 and c-Src. Kim et al. [25] tested the extract of Lycii Radicis Cortex (LRC) on an ovariectomized (OVX) rat model and they showed suppression of RANKL-induced osteoclast differentiation and also demonstrated inhibitory effect of LRC on NFATC1 expression. On the other hand, Kim et al. [26] showed that overexpression of NFATC1 induced differentiation of osteoclasts. Also, Zhao et al. [27] also showed that NFATC1 is an important transcription factor for RANKLmediated osteoclast differentiation, and Winslow et al. [28] demonstrated that lack of osteoclast differentiation and osteoporosis was observed on NFATC1 knock-out mice. Researchers also showed that inhibition of NFATC1 had an important role during the RANKL-induced osteoclast differentiation [29].

Many studies have shown the effects of inhibition of NFATC1 and FOS on osteoclasts and postmenopausal osteoporosis [8,20,29]. As we mentioned previously, FOS is another member of RANK/RANKL pathway and stimulation of FOS causes the activation of NFATC1 [11,27]. The FOS gene deletion was related to defective osteoclast differentiation and osteopetrosis in mice [25].

For many years, the interaction between NFATC1 and $F O S$ has been studied in vitro animal models conditions. Here, we studied NFATC1 and FOS methylation status in blood samples of post- and premenopausal women. In this study, we showed statistically significant association between postmenopause and unmethylation of the NFATC1 promotor $(p=0.010)$ and methylation of NFATC1 with premenopause. NFATC1 is important for osteoclast dif- 
ferentiation, which means important for bone resorption. According to our results, osteoporosis is not an expected condition in premenopausal women, and methylation data also confirms this hypothesis; in postmenopausal women, unmethylation of the NFATC1 promotor $(p=0.010)$ means differentiation of osteoclasts were triggered. These results could be used for epigenetic explanation of postmenopausal osteoporosis. Future studies should also be designed to inactivate the NFATC1 pathway as a possible targeted therapy strategy in postmenopausal osteoporosis. On the other hand, we did not find any significant associations between menopause and FOS methylation $(p>0.05)$. As far we know, epigenetic alterations are tissue-specific but we hope our results will shed light on further studies in this field, and encourage researchers to use bone and other related tissues for methylation analysis of this pathway. Up to now, there have been no epigenetic studies in this field, and we concluded that the NFATC1 gene was hypermethylated during the premenopausal period and it could be unmethylated during the postmenopausal period. We hope our results will lead epigenetic studies in this field.

Conclusions. In conclusion, we found significant association of unmethylation of NFATC1 and postmenopausal status. NFATC1 and osteoclast formation interaction has been shown by the in vitro studies. In this study, we concluded that epigenetic silencing of the NFATC1 gene can raise osteoclast activity and it is directly related to post-menopausal osteoporosis. These results will further epigenetic studies in postmenopausal women and could be used as a potential epigenetic marker during the evaluation of postmenopausal osteoporosis in clinical practice.

This study is the first epigenetic study that investigated the NFATC1 and FOS methylation in postmenopausal women and shows interaction between epigenetics and postmenopausal osteoporosis. Although the limited sample size in our study and lack of epigenetic studies in this field proves our results crucial and therefore, our results showed the magnitude of epigenetic profile of Turkish Cypriot postmenopausal women. Future studies in larger samples of postmenopausal women focused on the study of the different gene methylations will help to clarify potential effects of gene methylation in the menopause.

Acknowledgments. We thank all of the patients for their voluntary support. The authors also thank Dr. Pinar Tulay (Near East University, Nicosia, Turkish Republic of Northern Cyprus) and Mohammed Altarda for technical support and for help with DNA extraction. Authors' contribution: RK supervised the project, RK conceived and planned the experiments. OT carried out the statistical analysis. RK carried out the experiment. RK and OT wrote the manuscript. Both RK and OT contributed to the final version of the manuscript. Both authors provided critical feedback and helped shape the research, analysis and manuscript.

Declaration of Interest. The authors report no conflicts of interest. The authors alone are responsible for the content and writing of this article.

Funding. This study was supported by the Near East University Scientific Research Project Unit [Grant No. SAG-2016-2-012], Nicosia, Cyprus.

\section{REFERENCES}

1. López-Rodríguez C, Aramburu J, Jin L, Rakeman AS, Michino M, et al. Bridging the NFAT and NFkappaB families: NFAT5 dimerization regulates cytokine gene transcription in response to osmotic stress. Immunity. 2001; 15(1): 47-58.

2. Hogan PG, Chen L, Nardone J, Rao A. Transcriptional regulation by calcium, calcineurin, and NFAT. Genes Dev. 2003; 17(18): 2205-232.

3. Wu H, Peisley A, Graef IA, Crabtree GR. NFAT signaling and the invention of vertebrates. Trends Cell Biol. 2007; 17(6): 251-260.

4. Pan MG, Xiong Y, Chen F. NFAT gene family in inflammation and cancer. Curr Mol Med. 2013; 13(4): 543-554.

5. Takayanagi H, Kim S, Koga T, Nishina H, Isshiki M, Yoshida $\mathrm{H}$, et al. Induction and activation of the transcription factor NFATc1 (NFAT2) integrate RANKL signaling in terminal differentiation of osteoclasts. Dev Cell. 2002; 3(6): 889-901.

6. Matsumoto M, Kogawa M, Wada S, Takayanagi H, Tsujimoto M, Katayama S, et al. Essential role of p38 mitogen-activated protein kinase in cathepsin K gene expression during osteoclastogenesis through association of NFATc1 and PU.1. J Biol Chem. 2004; 279(44): 45969-45979.

7. Kim K, Kim JH, Lee J, Jin HM, Lee SH, Fisher DE, et al. Nuclear factor of activated T cells $\mathrm{c} 1$ induces osteoclast-associated receptor gene expression during tumor necrosis factor-related activation-induced cytokine-mediated osteoclastogenesis. J Biol Chem. 2005; 280(42): 35209-35216.

8. Chen S, Pan M. NFAT signaling and bone homeostasis. J Hematol Thromb Dis. 2013; 1(1): 102-108.

9. Kim JH, Kim N. Regulation of NFATc1 in osteoclast differentiation. J Bone Metab. 2014; 21(4): 233-241.

10. Huang H, Chang EJ, Ryu J, Lee ZH, Lee Y, Kim $\mathrm{HH}$. Induction of c-Fos and NFATc1 during RANKLstimulated osteoclast differentiation is mediated by 
the p38 signaling pathway. Biochem Biophys Res Commun. 2006; 351(1): 99-105.

11. Grigoriadis AE, Wang ZQ, Cecchini MG, Hofstetter W, Felix R, Fleisch HA, et al. c-Fos: A key regulator of osteoclast-macrophage lineage determination and bone remodeling. Science. 1994; 266(5184): 443-448.

12. Marini F, Cianferotti L, Brandi ML. Epigenetic mechanisms in bone biology and osteoporosis: Can they drive therapeutic choices? Int J Mol Sci. 2016; 17(8): 1329.

13. Wang J, Zhang W, Yu C, Zhang X, Zhang H, Guan $\mathrm{Q}$, et al. Follicle stimulating hormone increases the risk of postmenopausal osteoporosis by stimulating osteoclast differentiation. PLoS One. 2015; 10(8): e0134986.

14. Trošt Z, Trebše R, Preželj J, Komadina R, Logar DB, Marc J. A microarray based identification of osteoporosis-related genes in primary culture of human osteoblasts. Bone. 2010; 46(1): 72-80.

15. Ichikawa T, Horie-Inoue K, Ikeda K, Blumberg B, Inoue $\mathrm{S}$. Vitamin $\mathrm{K} 2$ induces phosphorylation of protein kinase $\mathrm{A}$ and expression of novel target genes in osteoblastic cells. J Mol Endocrinol. 2007; 39(4): 239-247.

16. Liu YZ, Dvornyk V, Lu Y, Shen H, Lappe JM, Recker $\mathrm{RR}$, et al. A novel pathophysiological mechanism for osteoporosis suggested by an in vivo gene expression study of circulating monocytes. J Biol Chem. 2005; 280(32): 29011-29016.

17. Leclerc N, Luppen CA, Ho VV, Nagpal S, Hacia JG, Smith E, et al. Gene expression profiling of glucocorticoid-inhibited osteoblasts. J Mol Endocrinol. 2004; 33(1): 175-193.

18. Chen XD, Xiao P, Lei SF, Liu YZ, Guo YF, Deng $\mathrm{FY}$, et al. Gene expression profiling in monocytes and SNP association suggest the importance of the STAT1 gene for osteoporosis in both Chinese and Caucasians. J Bone Miner Res. 2010; 25(2): 339-355.

19. Kalkan R, Altarda M, Tulay P, Tosun Ö. The interaction between ESRRA and PTH gene methylation and body mass index in post-menopausal cases. Cyprus J Med Sci. 2019; 4(3): 247-250.
20. Raje MM, Mhaske ST, Ghosh P, Wani RM, Ashma R. Differential gene expression pattern in osteoclast precursor cells of Indian postmenopausal women with and without osteoporosis: A microarray based study. J Bone Res. 2017; 5(3): 185-192.

21. Raggatt LJ, Partridge NC. Cellular and molecular mechanisms of bone remodeling. J Biol Chem. 2010; 285(33): 25103-25108.

22. Riggs BL, Khosla S, Melton LJ 3rd. Sex steroids and the construction and conservation of the adult skeleton. Endocr Rev. 2002; 23(3): 279-302.

23. Boroňová I, Bernasovská J, Mačeková S, Petrejčíková E, Tomková Z, Kl'oc J, et al. TNFRSF11B gene polymorphisms, bone mineral density, and fractures in Slovak postmenopausal women. J Appl Genet. 2015; 56(1): 57-63.

24. Gavali S, Gupta MK, Daswani B, Wani MR, Sirdeshmukh R, Khatkhatay MI. LYN, a key mediator in estrogen-dependent suppression of osteoclast differentiation, survival, and function. Biochim Biophys Acta Mol Basis Dis. 2019; 1865(3): 547-557.

25. Kim JH, Kim EY, Lee B, Min JH, Song DU, Lim JM, et al. The effects of Lycii Radicis Cortex on RANKLinduced osteoclast differentiation and activation in RAW 264.7 cells. Int J Mol Med. 2016; 37(3): 649658.

26. Kim K, Lee SH, Kim JH, Choi Y, Kim N. NFATc1 induces osteoclast fusion via up-regulation of Atp6v0d 2 and the dendritic cell-specific transmembrane protein (DC-STAMP). Mol Endocrinol. 2008; 22(1): 176-185.

27. Zhao Q, Wang X, Liu Y, He A, Jia R. FATc1: Functions in osteoclasts. Int J Biochem Cell Biol. 2010; 42(5): 576-579.

28. Winslow MM, Pan M, Starbuck M, Gallo EM, Deng L, Karsenty G, et al. Calcineurin/NFAT signaling in osteoblasts regulates bone mass. Dev Cell. 2006; 10(6): 771-782.

29. Xu J, Wu HF, Ang ESM, Yip K, Woloszyn M, Zheng $\mathrm{MH}$, et al. NF-kappaB modulators in osteolytic bone diseases. Cytokine Growth Factor Rev. 2009; 20(1): 7-17. 